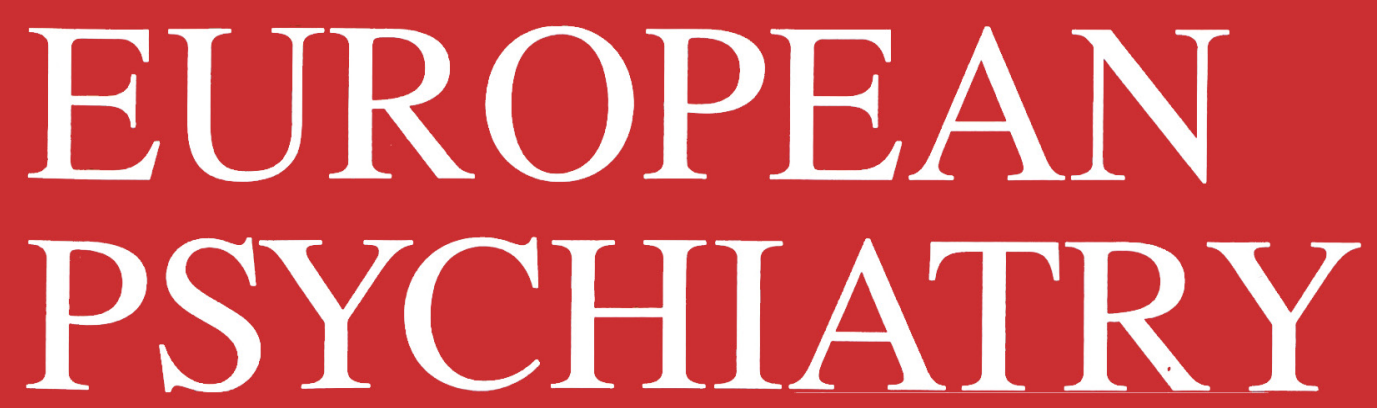

VOL $9 /$ No 4

1994

ISSN $0767-399 \times$ SCSPED II (1) $165-220$

PSYPEK (9) (4)

\title{
THE JOURNAL OF THE ASSOCIATION OF EUROPEAN PSYCHIATRISTS
}

\section{SPECIAL ARTICLE}

Placebo controlled studies in acute schizophrenia - an issue of concern

A Delini-Stula, D Berdah-Tordjman (Lingolsheim, France)

\section{ORIGINAL ARTICLES}

Incidence and comorbidity of personality disorders among first ever admitted psychiatric patients

O Mors, LV Sirensen (Risskov, Denmark)

Quantitative measurement of depression through speech analysis

JA Talavera, J Sáiz-Ruiz, M Garcia-Toro (Madrid, Spain)

Transcultural approach of depression. Comparative study of the diagnostic performance of the dexamethasone suppression test in São Paulo (Brazil) and Liège (Belgium)

LM Lucchesi, MH Fernandes, N Silva Junior, L Yazigi, M Ansseau, M Timsit-Berthier (São Paulo, Brazil; Liège, Belgium)

\section{SHORT COMMUNICATIONS}

Serum beta ${ }_{2}$-microglobulin as a possible early indicator of glomerular filtration impairment in subjects receiving long-term lithium treatment

G Rutigliano, F Vadruccio (Bari, Italy)

An objective method for edge detection in MRI morphometry

MS Keshavan, C Beckwith, W Bagwell, W Pettegrew, K Ranga Krishnan (Pittsburgh, Durham, USA)

\section{CASE REPORT}

Vitamin B12 deficiency presenting as dementia in a young adult: a case report

C Marino, B Grassi, F Macciardi, S Scarone (Milan, Italy)

\section{FOCUS ON EUROPEAN PRACTICE}

Influence of psychoanalytical training on professional performance in a sample of French speaking psychotherapists

A Dazord, P Gerin, JD Davis, ML Davis, N Aapro, J Thurin (Coventry, L'K; Paris, France) 
The journal of the Association of European Psychiatrists

Editors: C Ballus (Barcelona), JD Guelfi (Paris), H Heimann (Tübingen), R Murray (London)

Co-Editors: M Maj (Naples), CB Pull (Luxembourg), M Ackenheil (Munich), P Bech (Copenhagen)

Deputy editors: P Boyer, Y Lecrubier (Paris). Secretariat: Hôpital de la Salpêtrière, Pavillon Clérambault, 47, bd de l'Hôpital, 7565ı Paris Cedex 13, France. Tel: (0033) 1458670 22. Fax: (0033) 1 44239249

Advisory board

J Adès, Colombes, France

HS Akiskal, Rockville, USA

NC Andreasen, Iowa City, USA

J Angst, Zurich, Switzerland

P Baumann, Lausanne, Switzerland

H Beckmann, Würzburg, Germany

P Berner, Vienna, Austria

A Bertelsen, Risskov, Denmark

J Birley, London, UK

JC Bisserbe, Meudon, France

D Bobon, Glain-Liège, Belgium

B Bondy, Munich, Germany

JP Boulenger, Sherbrooke, Canada

F Brambilla, Milan, Italy

I Brockington, Birmingham, UK

A Clare, Dublin, Ireland

F Clerget-Darpoux, Paris, France

V Conde Lopez, Vallodolid, Spain

S Consoli, Paris, France

J Cottraux, Lyons, France

$M$ von Cranach, Kaufbeuren, Germany

A Dahl, Oslo, Norway

JM Danion, Sirasbourg, France

JFW Deakin, Manchester, UK

$\mathrm{M}$ de Bonis, Le Kremlin Bicêtre, France

H Dufour, Lausanne, Switzerland

R Engel, Munich, Germany

L Farde, Stockholm, Sweden

A Féline, Le Kremlin Bicêtre, France

A Fernandes da Fonseca, Porto, Portugal

F Ferrero, Geneva, Switzerland

M Fichter, Prien am Chiemsee, Germany

H Freeman, London, UK

\author{
HJ Gaertner, Tübingen, Germany \\ J Gibert, Cadiz, Spain \\ D Goldberg, Manchester, UK \\ I Hand, Hamburg, Germany \\ WE Haefely, Basel, Switzerland \\ H Häfner, Mannheim, Germany \\ T Helgason, Reykjavik, Iceland \\ H Hippius, Munich, Germany \\ A Jablenski, Sofia, Bulgaria \\ E Johnston, Edinburgh, UK \\ M Kastrup, Hvidovre, Denmark \\ D Kemali, Naples, Italy \\ R Kendell, Edinburgh, UK \\ D Klein, New York, USA \\ R Klein, New York, USA \\ S Langer, Paris, France \\ J Lellouch, Villejuif, France \\ T Lemperière, Colombes, France \\ J-P Lépine, Paris, France \\ OM Lesch, Vienna, Austria \\ SW Lewis, London, UK \\ H Lôo, Paris, France \\ JJ Lopez-Ibor, Madrid, Spain \\ P McGuffin, Cardiff, UK \\ W Maier, Mainz, Germany \\ A Mann, London, UK \\ K Mann, Tübingen, Germany \\ I Marks, London, UK \\ J Marlet, Venray, The Netherlands \\ J Massana Ronquillo, Barcelona, Spain \\ J Mendlewicz, Brussels, Belgium \\ HJ Möller, Bonn, Germany \\ D Naber, Munich, Germany
}

E O'Callaghan, Dublin, Ireland

Y Ono, Tokyo, Japon

M Patris, Strasbourg, France

J Pellet, Saint-Etienne, France

C Perris, Umeä, Sweden

P Pichot, Paris, France

H Pope, Belmont, USA

AJ Puech, Paris, France

G Racagni, Milan, Italy

N Retterstol, Oslo, Norway

M A Ron, London, UK

R Rosenberg, Risskov, Denmark

M Roth, Cambridge, UK

F Rouillon, Colombes, France

J Saiz-Ruiz, Madrid, Spain

A Sanchez-Blanque, Zaragoza, Spain

N Sartorius, Geneva, Switzerland

F Schulsinger, Copenhagen, Denmark

G Sedvall, Stockholm, Sweden

L Singer, Strasbourg, France

CN Stefanis, Athens, Greece

E Straube, Tübingen, Germany

E Strömgren, Risskov, Denmark †

E Taylor, London, UK

$P$ Taylor, London, UK

L Träskman-Bendz, Lund, Sweden

J Vallejo Ruibola, Barcelona, Spain

D Widlöcher, Paris, France

J Wilmotte, Marchienne du Pont, Belgium

J Wing, London, UK

FT Zimmer, Tübingen, Germany

J Zohar, Beer-Sheva, Israel

Association of European Psychiatrists

President: P Bech (Copenhagen); Past President: C Ballus (Barcelona); President Elect: R Murray (London); Secretary General: C Pull (Luxembourg); Treasurer: M Patris (Strasbourg); Counsellors: D Kemali (Naples), J Angst (Zurich); Section. L Singer (Strasbourg).

$\begin{array}{ll} & \text { EDITIONS SCIENTIFIQUES ELSEVIER } \\ & \text { 29, rue Buffon, F-75005 Paris } \\ & \text { Tel: (1) } 47071122-\text { Telecopier: (1) } 45871493 \\ & \text { A member of the Reed Elsevier ple group }\end{array}$

SUBSCRIPTIONS 1994 - Vol 9 in 6 issues

- France: $1380 \mathrm{FF}$ - Students: $828 \mathrm{FF}$ - EEC (organisms or firms; VAT registration number requested) and rest of the world: 1580 FF EEC (individuals) FF 1613 - Air delivery included.

Address order and payment to: Editions Scientifiques Elsevier

Bank: CCP Paris, No 3004100001 1904540H020/70.

- Subscriptions begin 4 weeks after receipt of payment and start with the first issue of the calendar year.

- Back issues and volumes are available from the publisher.

- Claims for missing issues should be made within six months of publication.

Consulting Translator: P Rousseau-Cunningham.

Advertising: Editions Scientifiques Elsevier (Anne Blanvillain).

(1) 1994 Editions Scientifiques Elsevier, Paris

All rights reserved. No part of this publication may be translated, reproduced, stored in a retrieval system or transmitted in any form or by any other means, electronic, mechanical, photocopying, recording or otherwise, without prior permission of the publisher.

No responsibility is assumed by the Publisher for any injury and/or damage to persons or property as a matter or products liability, negligence or otherwise, or from any use or operation of any methods, products, instructions or ideas contained in the material herein. Because of the rapid advances in the medical sciences, the Publisher recommends that independent verification of diagnoses and drug dosages should be made. 